\title{
Pectus excavatum, manejo mínimamente invasivo con técnica de Nuss. Experiencia Instituto Nacional del Tórax
}

\author{
Jorge Lavanderos F. ${ }^{1}$, Virginia Linacre S. ${ }^{2}$, Juan Emilio Cheyre F. ${ }^{2}$, \\ Jorge Armijo H. ${ }^{2}$ y Rafael Prats M. ${ }^{2}$
}

\section{Pectus excavatum, minimally invasive management with Nuss technique}

Aim: To communicate the experience in the treatment of pectus excavatum in the National Institute of Thorax. Materials and Method: Descriptive study with follow-up. Data of variables of interest were collected through the review of clinical records. Patients operated between March 2007 and April 2018 were included. Results: 86 patients operated with the Nuss technique were included in the period described. Of them, 74 male patients. The average age at the time of surgery was 17.8 years. The main reason for consultation was due to cosmetic detriment that corresponded to $41.8 \%$ of the patients. Except in 1 patient, in all the rest a single bar was occupied. In our series, there were 12 patients who presented some operative complication and 1 patient died. Up to the end of the follow-up, 61 bars were removed, 2 of them in non-scheduled surgery. There are 20 bars in situ and 4 patients of which there is no registration due to abandonment of controls. Discussion: Pectus excavatum is the most frequent of the deformities of the chest wall, it is 4 to 6 times more frequent in men than in women. In general, patients are asymptomatic, although some may have cardiopulmonary symptoms. The repair with minimally invasive surgery with Nuss technique appears today as the goldstandard of management. Conclusion: The management of patients with pectus excavatum in our Institution is similar to that reported in the international literature. Our efforts should aim to reduce the associated morbidity and mortality.

Key words: pectus excavatum; chest wall deformity; minimally invasive surgery.

\section{Resumen}

Objetivo: Comunicar la experiencia en el tratamiento de pectus excavatum en el Instituto Nacional del Tórax. Material y Método: Estudio descriptivo con seguimiento. Se recolectaron datos de las variables de interés, mediante la revisión de fichas clínicas. Se incluyeron pacientes operados entre marzo de 2007 y abril de 2018. Resultados: Se incluyeron 86 pacientes operados con técnica mínimamente invasiva en el período descrito. De ellos, 74 pacientes de sexo masculino. El promedio de edad al momento de la cirugía fue de 17,8 años. El principal motivo de consulta fue por desmedro estético que correspondió al $41,8 \%$ de los pacientes. Salvo en 1 paciente, en todo el resto se ocupó una sola barra. En nuestra serie hubo 12 pacientes que presentaron alguna complicación operatoria y 1 paciente fallecido. Hasta el cierre del seguimiento se retiraron 61 barras, 2 de ellas previo al período estipulado de tratamiento, por morbilidad. Hay 20 barras in situ y 4 pacientes de los cuales no se tiene registro por abandono de controles. Discusión: El pectus excavatum es la más frecuente de las deformidades de la pared torácica, es 4 a 6 veces más frecuente en hombres que en mujeres. En general los pacientes son asintomáticos, aunque algunos pueden presentar síntomas cardiopulmonares. La reparación con cirugía mínimamente invasiva con técnica de Nuss aparece hoy en día como el gold standard de manejo. Conclusión: El manejo de los pacientes con pectus excavatum en nuestra Institución se asemeja a lo reportado en la literatura internacional. Nuestros esfuerzos deben apuntar a disminuir la morbimortalidad asociada.

Palabras clave: pectus excavatum; deformidad de pared torácica; cirugía mínimamente invasiva.
'Residente de Cirugía de Tórax. Universidad de Chile. ${ }^{2}$ Cirujano de Tórax. Servicio Médico-Quirúrgico Respiratorio. Instituto Nacional del Tórax.

Recibido el 19 de enero de 2019 y aceptado para publicación el 26 de marzo de 2019.

Correspondencia a: Dr. Jorge Lavanderos F. jlavanderosfernandez@yahoo. com 


\section{Introducción}

El pectus excavatum es la anomalía más frecuente de la pared torácica, se ve más en hombres que en mujeres. La incidencia varía geográficamente y afecta aproximadamente a 1 de 400 individuos masculinos en el mundo occidental ${ }^{1}$.

Clásicamente, el pectus excavatum se consideraba un problema estético para los pacientes, pero estudios recientes han demostrado que el gasto cardíaco puede reducirse hasta en un $20 \%$, afectándose también la capacidad ventilatoria y la movilidad torácica; lo que genera una menor tolerancia al ejercicio, síntoma referido hasta en el $60 \%$ de los pacientes.

El objetivo de la corrección de la deformidad del tórax es permitir un normal desarrollo de la función cardiopulmonar y corregir un defecto estético que genera un importante desmedro en la autoestima de los pacientes ${ }^{1}$.

La reparación mínimamente invasiva del pectus excavatum se realizó por primera vez en 1987 y en 1998; Nuss et al., publicaron su experiencia de 10 años de tratamiento. Dicha técnica cambió el enfrentamiento quirúrgico hacia estos pacientes, considerándose actualmente este abordaje como el tratamiento de elección para su corrección ${ }^{2}$.

La técnica consiste en la instalación de una barra metálica retroesternal bajo visión videotoracoscópica con lo que se corrige el defecto. La cirugía requiere anestesia general e intubación de doble lumen, paciente en posición decúbito supino con ambos brazos en abducción, se marca el sitio de mayor declive de la deformidad que es por donde pasará la barra. Se tallan bolsillos subcutáneos bilaterales en la línea axilar anterior para introducir el sable de disección que pasa de derecha a izquierda, la barra seleccionada según el tamaño del paciente se curva proporcional a la deformidad a corregir. La barra se fija con una placa estabilizadora en el bolsillo izquierdo y con sutura irreabsorbible en el lado derecho. En algunas ocasiones, como reoperaciones o según la preferencia del cirujano, también se fija con placa estabilizadora a derecha. De rutina no se instalan drenes pleurales. Las ventajas de la técnica en relación con las técnicas abiertas son prescindir de grandes incisiones, no se necesitan colgajos, ni osteotomías o resecciones condrocostales, menor tiempo operatorio, menor sangrado y los resultados estéticos son muy satisfactorios ${ }^{3}$.

El objetivo de nuestro trabajo es comunicar la experiencia en su tratamiento con técnica mínimamente invasiva.

\section{Materiales y Métodos}

Estudio descriptivo con seguimiento. Se recolectaron datos de las variables de interés mediante la revisión de fichas clínicas. Se incluyeron pacientes con diagnóstico de pectus excavatum operados entre marzo de 2007 y abril de 2018. No se incluyeron pacientes corregidos con técnica de Ravitch (cirugía abierta que incluye resección de cartílagos costales y osteotomía esternal) ${ }^{4}$. El seguimiento se realizó en policlínico de cirugía de tórax del Instituto Nacional del Tórax hasta noviembre de 2018.

Dentro de las variables analizadas se consideró edad, sexo, motivo de consulta, características clínicas del pectus, índice de Haller, estudio preoperatorio, días desde la cirugía hasta el alta, longitud de la barra, uso de estabilizador, presencia de complicaciones (dentro de los 30 días posteriores a la cirugía), manejo de las complicaciones, mortalidad y tiempo hasta el retiro de la barra.

Para el análisis de datos se utilizó el programa MS Excel, la evaluación de la información fue del tipo descriptiva considerando los datos como variables cuantitativas expresadas en números y porcentajes.

\section{Resultados}

Se incluyeron 86 pacientes operados con técnica de Nuss en el período descrito. De ellos, 74 de sexo masculino. El promedio de edad al momento de la cirugía fue de 17,8 años (14-32). En nuestra casuística hay 3 pacientes con síndrome de Marfán y 1 paciente con síndrome de Williams. El principal motivo de consulta fue por desmedro estético que correspondió al 41,8\% de los pacientes, seguido de la disnea con un $19,7 \%$. Más de la mitad del total de pacientes refieren una baja en su autoestima. En la evaluación clínica en $41(47,6 \%)$ pacientes la deformidad era simétrica, en $9(10,4 \%)$ asimétrica y en 36 casos no fue registrado (Figura 1). En 50 pacientes se registró el índice de Haller, cuyo promedio fue de 3,8 (1,4-7,6); en 36 pacientes el índice fue mayor a 3,25 , sin considerarse para la indicación quirúrgica. Con respecto al estudio preoperatorio, todos nuestros pacientes se realizaron tomografía computada de tórax, hubo $69(80,2 \%)$ pacientes con ecocardiografía y $61(70,9 \%)$ con espirometría (43\% con patrón restrictivo); hubo un paciente que además se estudió con resonancia magnética de tórax. La estancia hospitalaria posterior a la cirugía hasta el alta fue en promedio 5,7 días (3-14). En 48 pacientes se registró la longitud de la barra, la más utilizada fue 
la de 14 pulgadas. Salvo en un paciente que se ocuparon 2 barras, en todo el resto se ocupó sólo una. En un solo caso se utilizó estabilizador bilateral y en un solo caso el estabilizador se instaló a derecha. En 5 pacientes se instaló pleurostomía al término de la cirugía por preferencia del cirujano, sin haber tenido ningún incidente quirúrgico intraoperatorio. En nuestra serie hubo 12 (14,1\%) pacientes que presentaron alguna complicación operatoria, de ellas 5

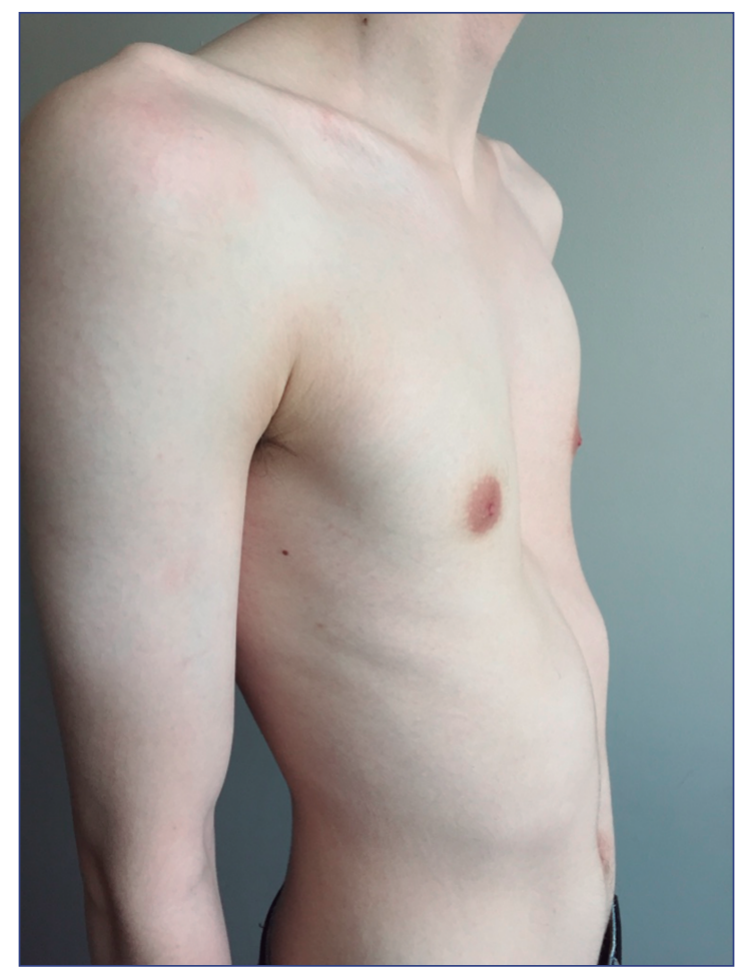

Figura 1. Pectus excavatum simétrico. consideradas como complicaciones menores según la Clasificación de Clavien-Dindo. Hubo 1 paciente fallecido por lesión miocárdica al momento de la instalación de la barra que fallece 10 días después de la cirugía por falla multiorgánica (Tabla 1). Hasta el cierre del seguimiento se retiraron 59 barras, con tiempo promedio de permanencia de 39,3 meses (24-80), más 2 casos en que hubo retiro previo al tiempo mínimo de tratamiento por complicaciones tardías. Hay 20 barras in situ con tiempo promedio de permanencia de 31,5 meses (7-51) y 4 pacientes de los cuales no se tiene registro por abandono tardío de controles.

\section{Discusión}

La reparación del pectus excavatum mediante abordaje mínimamente invasivo ha reportado buenos resultados. Las indicaciones para la reparación quirúrgica incluyen compresión cardíaca, intolerancia al ejercicio o insatisfacción cosmética ${ }^{5}$. El principal motivo de consulta registrado en nuestra serie fue por estética lo que refleja la importancia de la imagen corporal en nuestra relación con la sociedad, en especial considerando que gran parte de los pacientes intervenidos son adolescentes. En sólo 36 pacientes se registró un índice de Haller mayor a 3,25 . Inicialmente este índice fue establecido como el estándar para determinar la gravedad del pectus; sin embargo, este índice puede no reflejar con precisión la gravedad de la malformación, debido a que este es una relación del diámetro transversal del tórax con el diámetro anteroposterior en su punto más estrecho, lo que varía de acuerdo a la conformación del tórax ${ }^{5,6}$. El estudio preoperatorio de nuestros

Tabla 1. Complicaciones

\begin{tabular}{|c|c|c|c|}
\hline Complicación & $\mathrm{N}^{0}$ pacientes & Manejo & $\begin{array}{l}\text { Clasificación de } \\
\text { clavien-dindo }\end{array}$ \\
\hline Neumotórax & 4 & Pleurostomía & IIIa \\
\hline \multirow[t]{2}{*}{ Infección de herida } & 1 & Retiro de fijador + aseo + VAC & $\mathrm{IIIb}$ \\
\hline & 1 & Curaciones & I \\
\hline Hematoma de pared & 2 & Manejo conservador & I \\
\hline Derrame pleural leve & 1 & Antibióticos empíricos & II \\
\hline Dehiscencia + infección de herida + derrame pleural & 1 & Retiro de barra + pleurostomía + aseos & $\mathrm{IIIb}$ \\
\hline Infección de herida + fiebre & 1 & Rehospitalización + curaciones + antibióticos & II \\
\hline Desplazamiento de la barra & 1 & Reoperación al día siguiente & $\mathrm{IIIb}$ \\
\hline Lesión miocárdica & 1 & Manejo en UCI & $\mathrm{V}$ \\
\hline
\end{tabular}


pacientes se realizó con una tomografía computada (TC) de tórax, una ecococardiografía transtorácica y una espirometría. La TC de tórax proporciona una imagen más clara de la deformidad torácica, permite descartar otras anomalías anatómicas intratorácias y permite la planificación quirúrgica. La reconstrucción tridimensional es muy útil para determinar el número de barras que serán necesarias para la corrección ${ }^{7}$. Del mismo modo, las compresiones cardíacas y pulmonares, así como la relación del esternón con el corazón comprimido se visualizan muy bien en la TC. La evaluación cardiológica es importante porque un número significativo de pacientes tiene hallazgos de compresión auricular y ventricular derecha, prolapso de la válvula mitral y anomalías del ritmo; además, es imprescindible descartar patología cardíaca que requiera ser corregida previa a la cirugía de pectus ${ }^{4,7}$. Los resultados de la espirometría suelen ser entre un 10 y un $20 \%$ menos que el promedio esperado para la población, con valores compatibles con enfermedad restrictiva en un número no despreciable de pacientes ${ }^{2}$, tal como se evidenció en nuestra casuística.

En la mayoría de los pacientes se instaló una sola barra y un solo estabilizador, lo que concuerda con lo realizado y publicado en series internacionales ${ }^{6}$ (Figura 2). En nuestra serie hubo 12 (14,1\%) pacientes que presentaron alguna complicación operatoria; las más frecuentes el neumotórax y la infección de herida operatoria, con $4(4,7 \%)$ casos cada una. Las complicaciones reportadas en series internacionales de centros con alto volumen de pacientes son prin-

Figura 2. Barra única con estabilizador unilateral a izquierda. cipalmente neumotórax, desplazamiento de la barra, infección de herida operatoria, hemotórax y exposición de la barra por dehiscencia de la herida. El neumotórax generalmente se resuelve espontáneamente y es realmente parte de la operación en lugar de una verdadera complicación, lo que se previene poniendo especial cuidado en la insuflación pulmonar al término de la cirugía. El manejo con pleurostomía del neumotórax posterior a la reparación del pectus no debería ser mayor al 3\% de los pacientes. Esta es una cirugía que se clasifica como una herida limpia, por lo que la tasa de infección de la herida debería ser menor al $1 \%$, valores que en nuestros resultados se ven superados ${ }^{2}$.

El desplazamiento de la barra es una de las complicaciones más temidas. La tasa de desplazamiento inicial reportada fue de $18,5 \%$, después de la introducción de los estabilizadores, la tasa bajó a 7,4\%, y con la adición de suturas pericostales colocadas alrededor de la barra y de las costillas subyacentes, la tasa bajó al 1 a $2 \%$.

La penetrante cardíaca, que fue la causa de mortalidad de uno de nuestros pacientes, es una de las complicaciones más temidas de la técnica y descrita en la literatura principalmente cuando la técnica en sus inicios no se realizaba bajo visión toracoscópica, tal como lo describe Nuss en sus publicaciones sobre el desarrollo y evolución de la técnica quirúrgica $^{2}$. La revisión de la TC preoperatoria para determinar la posición del corazón y su relación con el esternón es útil para planificar el procedimiento, especialmente en pacientes con asimetría severa y rotación esternal ${ }^{2}$. Si el corazón está muy comprimido, se puede elevar el esternón con un gancho o una ventosa durante la tunelización, minimizando el riesgo de lesiones, de gran importancia es que la punta del sable siempre debe mantenerse a la vista (Figura 3). La buena visibilidad con el toracoscopio

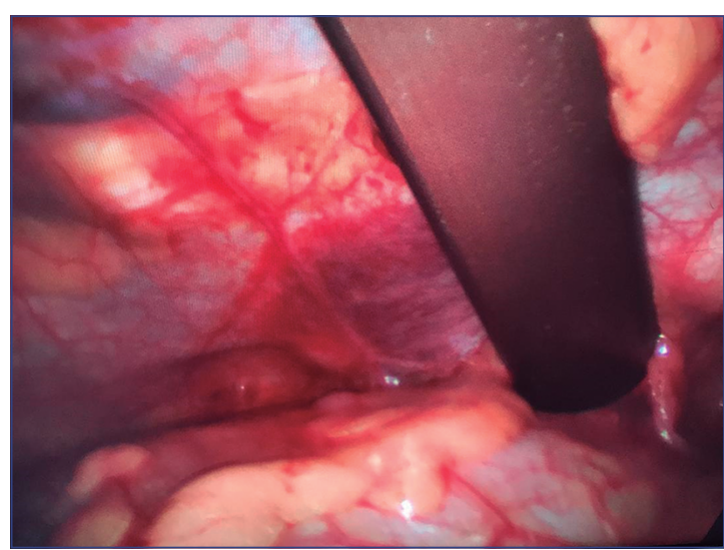

Figura 3. Paso retroesternal del sable, visión toracoscópica. 
es esencial y, si es necesario, debe utilizarse toracoscopía bilateral ${ }^{5}$.

El tiempo promedio de permanencia de la barra en nuestros pacientes fue de 39,3 meses (3,2 años). Las barras deben permanecer en el tórax durante 2 a 4 años después de la reparación del pectus. La mayoría de los pacientes toleran bien la barra durante 3 años y pueden participar en deportes competitivos con la barra in situ. Si la barra se retira antes de 2 años, la tasa de recurrencia aumenta inversamente con el tiempo que la barra permanece en su lugar ${ }^{8}$.

Es importante señalar las limitaciones de nuestro trabajo, si bien el número de pacientes para la realidad nacional es bastante considerable, comparado con publicaciones internacionales puede considerarse bajo. Por otra parte, el registro incompleto de datos en las fichas clínicas es una limitante en la valorización cuantitativa para el análisis de las variables de interés.

\section{Conclusión}

El manejo de los pacientes con pectus excavatum en nuestra Institución se asemeja a lo reportado en la literatura internacional. Nuestros esfuerzos deben apuntar a disminuir la morbimortalidad asociada.

\section{Responsabilidades éticas}

Protección de personas y animales. Los autores declaran que para esta investigación no se han realizado experimentos en seres humanos ni en animales.

Confidencialidad de los datos. Los autores declaran que en este artículo no aparecen datos de pacientes.

\section{Conflicto de Intereses: Ninguno.}

\section{Referencias}

1. Pilegaard H, Bjorn Licht P. Minimal Invasive Repair of Pectus Excavatum and Carinatum. Thorac Surg Clin. 2017;27:123-31.

2. Nuss D, Kelly Jr R. Indications and Technique of Nuss Procedure for Pectus Excavatum. Thorac Surg Clin. 2010;20 583-97.

3. Prats R, González R, Lazo D, Venturelli F, Santolaya R, Rodríguez P. Operación de Nuss. Corrección mínimamente invasiva del Pectus excavatum en adultos Rev Chil
Cir. 2010;62:309-13.

4. Kelly R. Pectus excavatum: historical background, clinical picture, preoperative evaluation and criteria for operation. Seminars in Pediatric Surgery 2008;17:181-93.

5. Poston P, Patel S, Rajput M, Rossi N, MD, Ghanamah M, Davis J, Turek J. The Correction Index: Setting the Standard for Recommending Operative Repair of Pectus Excavatum. Ann Thorac Surg. 2014;97:1176-80.

6. Sesia S, Heitzelmann M, Schaedelin S, Magerkurth O, Kocher G, Schmid R, et al. Standardized Haller and Asymmetry Index Combined for a More Accurate Assessment of Pectus Excavatum. Ann Thorac Surg. 2018;107:1-6. DOI: 10.1016/j.athoracsur.2018.07.086.

7. Zhang D, Tang J, Ben X, Xie L, Zhou $\mathrm{H}$, Xiong Ye, et al. Surgical correction of 639 pectus excavatum cases via the Nuss procedure. J Thorac Dis. 2015;7:1595605.

8. Gon Cho D, Kim J, Park J, Moon S. Recurrence of pectus excavatum following the Nuss procedure. J Thorac Dis. 2018;10:6201-10. 